\title{
Synthesis of $\mathrm{MoSe}_{2} / \mathrm{SrTiO}_{3}$ Heterostructures with Enhanced Ultraviolet-Light-Driven and Visible-Light-Driven Photocatalytic Properties
}

\author{
Xingwang Zhou, Jun Yao, Ming Yang, Jiangquan Ma*, Qingwei Zhou, \\ Ershen $\mathrm{Ou}$, Zhen Zhang and Xueni Sun \\ Advanced Catalysis and Green Manufacturing \\ Collaborative Innovation Center, Changzhou University \\ Changzhou 213164, P. R. China \\ *majiangquan@126.com
}

Received 11 January 2018

Accepted 15 March 2018

Published 11 April 2018

\begin{abstract}
In this research, a series of novel $\mathrm{MoSe}_{2} / \mathrm{SrTiO}_{3}$ heterostructures were successfully prepared by a two-step hydrothermal method. The samples were characterized by XRD, UV-Vis, SEM, TEM, EDS and XPS. Results showed that the degradation of MO under UV is better than that under the visible light. And, $\mathrm{MoSe}_{2}$ loaded on $\mathrm{SrTiO}_{3}$ under UV irradiation demonstrated a higher catalytic activity. The degradation rate of methyl orange was $99.46 \%$ for $\mathrm{MoSe}_{2} / \mathrm{SrTiO}_{3}$ under the optimum loading weight $(0.1 \mathrm{wt} . \%)$. This is mainly because the combination of $\mathrm{MoSe}_{2}$ and $\mathrm{SrTiO}_{3}$ prevents electrons and holes recombination in $\mathrm{SrTiO}_{3}$ and $\cdot \mathrm{O}_{2}^{-}$appears in the system. In general, $\mathrm{MoSe}_{2} / \mathrm{SrTiO}_{3}$ heterostructures have good environmental friendliness for photocatalytic degradation.
\end{abstract}

Keywords: Two-step hydrothermal method; $\mathrm{MoSe}_{2} / \mathrm{SrTiO}_{3}$; photocatalytic degradation; methyl orange.

\section{Introduction}

New renewable energies have been explored to replace traditional fossil fuels. And, development of semiconductors has become a hot spot. ${ }^{1}$ Among those semiconductors, perovskite oxide is widely used as a photocatalyst in photodegradation and light hydrogen production because of its stable chemical structure and nontoxicity. ${ }^{2}$

Perovskite oxide generally refers to the oxide with $\mathrm{ABO}_{3}$ or $\mathrm{A}_{2} \mathrm{BO}_{4}$ structure. ${ }^{3}$ In the $\mathrm{ABO}_{3}$ structure, $\mathrm{A}$ is a rare earth metal with a large ionic radius, $\mathrm{B}$ is a transition metal with a small ionic radius. $\mathrm{A}_{2} \mathrm{BO}_{4}$ structure consists of $\mathrm{ABO}_{3}$ and $\mathrm{AO}$ layers alternately. ${ }^{4} \mathrm{In}$ this frame, $\mathrm{A}$ is a larger cation at the edge of the structure, and $\mathrm{B}$ is a smaller cation located in the center octahedron. ${ }^{5}$ Among the perovskite oxides, strontium titanate $\left(\mathrm{SrTiO}_{3}\right)$ and strontium zirconate $\left(\mathrm{SrZrO}_{3}\right)$ are representative. ${ }^{6}$ The decomposition of water into hydrogen and oxygen is a nonspontaneous reaction. Thermodynamics requires that the conduction band and valence band position of the semiconductor photocatalyst must be matched with the reduction of water and the oxidation potential. That is to say, the

${ }^{*}$ Corresponding author. 
conduction band of the semiconductor is lower than the $\mathrm{H}^{+} / \mathrm{H}_{2}(0 \mathrm{~V}$ versus NHE) level, the valence band is higher than the $\mathrm{O}_{2} / \mathrm{H}_{2} \mathrm{O}(1.23 \mathrm{~V})$. Thus, $\mathrm{SrTiO}_{3}$ and $\mathrm{SrZrO}_{3}$ are usually used to degrade dyes and decompose $\mathrm{H}_{2} \mathrm{O}$ into $\mathrm{H}_{2}$ and $\mathrm{O}_{2} \cdot{ }^{7,8}$ Liu et al. proved that the $\mathrm{MoS}_{2} / \mathrm{SrTiO}_{3}$ composite material with 0.05 wt.\% of $\mathrm{MoS}_{2}$ showed the best photocatalytic activity, with a MO degradation rate as high as $99.81 \%$ after $60 \mathrm{~min}$ UV irradiation time. ${ }^{9}$ Tian et al. observed that the $\mathrm{MoS}_{2} / \mathrm{SrZrO}_{3}$ heterostructure with an optimal content of $0.05 \mathrm{wt} . \%$ $\mathrm{MoS}_{2}$ exhibited the highest $\mathrm{H}_{2}$ evolution rate of $5.31 \mathrm{mmol} \mathrm{h}^{-1} .{ }^{10}$ Recently, studies have been carried out on $\mathrm{SrTiO}_{3}$ based materials, including pure $\mathrm{SrTiO}_{3}, \mathrm{SrTiO}_{3}$ supported heterostructures, metal ions doped $\mathrm{SrTiO}_{3}$ and carbon nitride $\mathrm{SrTiO}_{3}$ heterostructures. ${ }^{11-13}$

Each Mo atom of $\mathrm{MoSe}_{2}$ is surrounded by six Se atoms to form a triangular prism. ${ }^{14}$ Mo-Se also has many edges, resulting in a large specific surface area and excellent catalytic activity. ${ }^{15,16}$ At the same time, $\mathrm{MoSe}_{2}$ belongs to the transition metal chalcogenides. ${ }^{17}$ Also, the band of $\mathrm{MoSe}_{2}$ is narrow with great stability, which helps to improve the photocatalytic efficiency of $\mathrm{SrTiO}_{3}$ support. ${ }^{18}$ This is important for solving energy shortages and environmental pollution.

In this study, a series of $\mathrm{MoSe}_{2} / \mathrm{SrTiO}_{3}$ heterostructures were synthesized by a simple two-step hydrothermal method. The photocatalytic performances in the degradation of methyl orange (MO) were investigated under both UV and visible light conditions. Research results indicated that $\mathrm{MoSe}_{2}$ can transfer the metal properties of electrons and improve the photocatalytic activity of $\mathrm{SrTiO}_{3}$ under UV irradiation. To the best of our knowledge, this is the first attempt to prepare the $\mathrm{MoSe}_{2} / \mathrm{SrTiO}_{3}$ heterostructures and apply them to the MO degradation system under UV light and visible light irradiation.

\section{Materials and Methods}

\subsection{Chemicals and materials}

Citric acid monohydrate $\left(\mathrm{C}_{6} \mathrm{H}_{8} \mathrm{O}_{7} \cdot \mathrm{H}_{2} \mathrm{O}, 99.5 \%\right)$, tetraisopropyl orthotitanate $\left(\mathrm{C}_{12} \mathrm{H}_{28} \mathrm{O}_{4} \mathrm{Ti}, 99.9 \%\right)$, strontium nitrate $\left(\mathrm{Sr}\left(\mathrm{NO}_{3}\right)_{2}, 99.5 \%\right)$, sodium hydroxide $(\mathrm{NaOH}, 97.0 \%)$, sodium molybdate $\left(\mathrm{Na}_{2} \mathrm{MoO}_{4} \cdot 2 \mathrm{H}_{2} \mathrm{O}, 99.0 \%\right)$, selenium powder (Se, $99.0 \%)$, sodium borohydride $\left(\mathrm{NaBH}_{4}, 96.0 \%\right)$, MO
(96.0\%), acetic acid $\left(\mathrm{CH}_{3} \mathrm{COOH}, 99.8 \%\right)$ and anhydrous ethanol $\left(\mathrm{C}_{2} \mathrm{H}_{5} \mathrm{OH}, 99.7 \%\right)$ were all purchased from the Sinopharm Group Chemical Reagent Co., Ltd. in analytical grade, and were used as received without further purification. Deionized water was used throughout the whole experiment.

\subsection{Synthesis of $\mathrm{SrTiO}_{3}$}

$\mathrm{C}_{6} \mathrm{H}_{8} \mathrm{O}_{7} \cdot \mathrm{H}_{2} \mathrm{O}$ (1.26g) was dissolved in ethanol $(15 \mathrm{~mL})$, followed by adding $\mathrm{C}_{12} \mathrm{H}_{28} \mathrm{O}_{4} \mathrm{Ti}(1.5 \mathrm{~mL})$ to form solution 1 . Solution 2 was prepared by dissolving $\operatorname{Sr}\left(\mathrm{NO}_{3}\right)_{2}$ (1.06g) in deionized water $(15 \mathrm{~mL}) . \mathrm{NaOH}(2 \mathrm{~g})$ was dissolved in deionized water $(15 \mathrm{~mL})$ to form solution 3 . The solution 2 and 3 were separately added to solution 1 with mechanical stirring for $30 \mathrm{~min}$. Then, the mixed solution was sealed in a Teflon-lined stainless-steel autoclave. The autoclave was cooled down to room temperature, followed by centrifugation. The product obtained was washed three times with deionized water, acetic acid and ethanol. The product was vacuum-dried at $80^{\circ} \mathrm{C}$ for $24 \mathrm{~h}$ to obtain $\mathrm{SrTiO}_{3}$.

\subsection{Synthesis of $\mathrm{MoSe}_{2} / \mathrm{SrTiO}_{3}$ heterocatalyst}

$1 \mathrm{~g}$ as-prepared $\mathrm{SrTiO}_{3}$ was dissolved in the mixture containing $30 \mathrm{~mL}$ distilled water and $30 \mathrm{~mL}$ ethanol, a certain quality $\mathrm{Na}_{2} \mathrm{MoO}_{4} \cdot 2 \mathrm{H}_{2} \mathrm{O}$, Se, $\mathrm{NaBH}_{4}$ were then added into the solution. The obtained suspension was stirred for $30 \mathrm{~min}$ for uniform dispersion. The mixed solution was then sealed in a PPLlined stainless-steel autoclave. The autoclave was heated to $240^{\circ} \mathrm{C}$ for $48 \mathrm{~h}$. After cooling to the room temperature, the product was separated by centrifugation, washed with distilled water and ethanol three times. Finally, the catalysts were dried in vacuum at $80^{\circ} \mathrm{C}$ for $24 \mathrm{~h}$. The prepared samples with $0.01,0.05,0.1,0.5,1,3,5$ wt. $\% \mathrm{MoSe}_{2}$, defined as the weight ratios of $\mathrm{MoSe}_{2}$ to $\mathrm{SrTiO}_{3}$, were named as S1, S2, S3, S4, S5, S6, S7.

\subsection{Photocatalytic activity test}

$10 \mathrm{mg}$ MO was dissolved in $500 \mathrm{~mL}$ deionized water to obtain MO dye solution with a concentration of $20 \mathrm{mg} \mathrm{L}^{-1}$. $0.2 \mathrm{~g}$ photocatalyst was added into MO dye solution and stirred for $30 \mathrm{~min}$ in the absence of light to reach the adsorption equilibrium. The initial concentration of the mixed solution was designated as $C_{0}$. Experiments were carried out to evaluate the 
photocatalytic activity. Under UV-light and visible light irradiation, $4 \mathrm{~mL}$ of the liquid sample was periodically extracted every $15 \mathrm{~min}$. Then the photocatalyst and the solution were separated by centrifugation. Finally, the absorbance of the filtrates was measured by a spectrophotometric technique. The photocatalytic performance was measured by the dye removal rate, which is calculated by the following formula:

$$
D=\frac{C_{0}-C}{C_{0}} \times 100 \%,
$$

where $D(\%)$ is the degradation rate, $C_{0}\left(\mathrm{mg} \mathrm{L}^{-1}\right)$ denotes the initial MO dye concentration, and $C\left(\mathrm{mg} \mathrm{L}^{-1}\right)$ represents the MO dye concentration in the filtrates after irradiation.

\subsection{Characterization of photocatalyst}

$\mathrm{D} / \max 2500 \mathrm{PC} \mathrm{X}$-ray diffractometer was used to analyze photocatalysts $(\mathrm{CuK} \alpha$ as the radiation source, $\lambda=1.54056 \AA$ ). Scanning electron microscopy (SEM, JEOL/JSM-6360LA) and transmission electron microscopy (TEM, JEM-2010) were used to observe the morphology and other internal microstructure of photocatalysts. The elements and its valence were analyzed by X-ray photoelectron spectroscopy (XPS, Thermo-250XI) and energy dispersive spectrometer (EDS, Ametek Genesis$\mathrm{XM260S).} \mathrm{UV-Vis} \mathrm{absorption} \mathrm{spectra} \mathrm{were} \mathrm{mea-}$ sured on a Hitachi U-4100 spectrometer.

\section{Results and Discussion}

The XRD patterns of the catalysts are shown in Fig. 1. All the peaks were assigned as cubic perovskite structure (JCPDS card 35-0734) at $2 \theta$ with angles of $32.4^{\circ} \mathrm{C}, 39.9^{\circ} \mathrm{C}, 46.5^{\circ} \mathrm{C}, 57.7^{\circ} \mathrm{C}, 67.6^{\circ} \mathrm{C}$ and $77.1^{\circ} \mathrm{C}$ corresponding to the indices of the (110), (111), (200), (211), (220) and (310) planes. However, no obvious peaks of heterojunctions attributed to $\mathrm{MoSe}_{2}$ phase were observed in S1-S7 samples. This is mainly due to the low load, uniform distribution and poor crystallization of $\mathrm{MoSe}_{2}$. The diffraction peaks of S1-S7 were similar to pure $\mathrm{SrTiO}_{3}$ indicating that no new phase of $\mathrm{SrTiO}_{3}$ was presented in $\mathrm{MoSe}_{2} / \mathrm{SrTiO}_{3}$.

The morphology of $\mathrm{SrTiO}_{3}$ is a nanosphere [in Figs. 2(a), 2(b) and Figs. 3(a), 3(b)]. From Figs. 2(c) and 2(d), the flower-like $\mathrm{MoSe}_{2}$ is loaded on the spherical $\mathrm{SrTiO}_{3}$ surface. Therefore, it can be

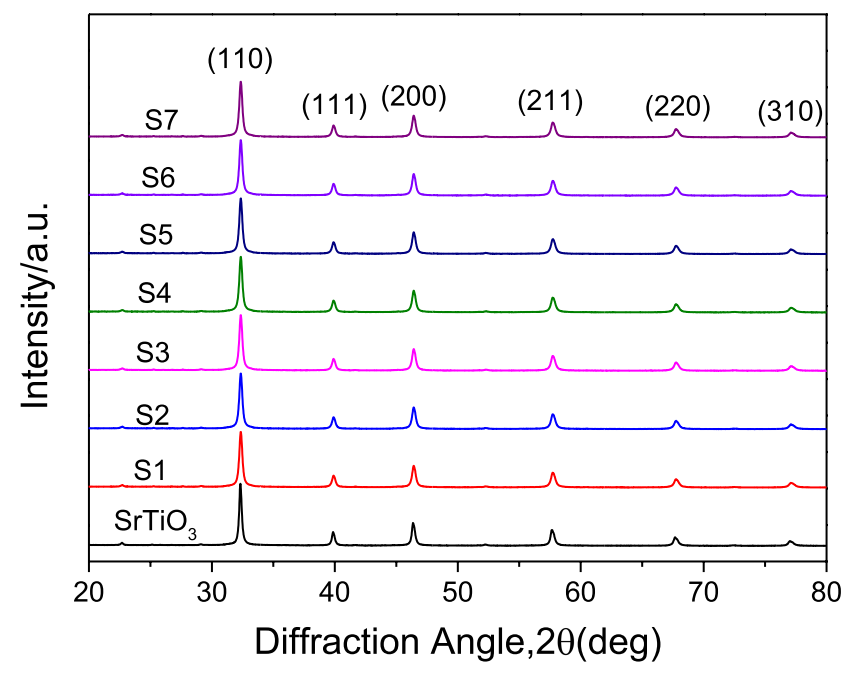

Fig. 1. XRD patterns of $\mathrm{SrTiO}_{3} \mathrm{~S} 1, \mathrm{~S} 2, \mathrm{~S} 3, \mathrm{~S} 4, \mathrm{~S} 5, \mathrm{~S} 6, \mathrm{~S} 7$.

proved indirectly that $\mathrm{MoSe}_{2} / \mathrm{SrTiO}_{3}$ heterostructure is successfully synthesized. The mechanism of $\mathrm{MoSe}_{2}$ loading on $\mathrm{SrTiO}_{3}$ surface was illustrated in Fig. 2(e). Figure 3(e) shows the presence of Sr, Ti, O, Mo and Se elements for the EDS of S3 samples. Based on the HRTEM of S3 in Figs. 3(c) and $3(\mathrm{~d})$, the lattice spacing was calculated to be $0.65 \mathrm{~nm}$, corresponding to the (002) plane of $\mathrm{MoSe}_{2}$, indicating that $\mathrm{MoSe}_{2}$ was successfully loaded on $\mathrm{SrTiO}_{3}$ surface. As a result, $\mathrm{MoSe}_{2} / \mathrm{SrTiO}_{3}$ heterostructures were successfully prepared.

Based on the Kubelka-Munk theory, the UV-Vis of $\mathrm{SrTiO}_{3}$ shows a significant steep absorption edge located at about $392 \mathrm{~nm}$ which can be assigned to its intrinsic bandgap absorption (in Fig. 4). The energy band of $\mathrm{SrTiO}_{3}$ is calculated to be $3.2 \mathrm{eV}$ according to the formula $E(\mathrm{eV})=1240 / \lambda(\mathrm{nm}) .{ }^{19}$ The UV-Vis of S1, S2, S3, S4, S5, S6, S7 are similar to $\mathrm{SrTiO}_{3}$ and are in the range of ultraviolet light. The UV absorption edge of $\mathrm{MoSe}_{2}$ is at $710 \mathrm{~nm}$, so the energy band of $\mathrm{MoSe}_{2}$ is $1.75 \mathrm{eV} .^{20,21}$

No obvious spectrum difference was caused by $\mathrm{MoSe}_{2}$ loading. Thus, the elements of sample S7 was investigated by XPS as shown in Fig. 5. Figure 5(a) shows a comparison of XPS for $\mathrm{SrTiO}_{3}$ and $\mathrm{S} 7$ samples. The compositions of $\mathrm{Sr}$, Ti and $\mathrm{O}$ elements for $\mathrm{SrTiO}_{3}$ and $\mathrm{S} 7$ are shown in Figs. 5(b)-5(d). After the $\mathrm{MoSe}_{2}$ was supported to $\mathrm{SrTiO}_{3}$, a higher binding energy of Sr $3 \mathrm{~d}$, Ti $2 \mathrm{p}$ and $\mathrm{O}$ 1s of $\mathrm{MoSe}_{2} / \mathrm{SrTiO}_{3}$ shifted by about $0.25 \mathrm{eV}$ can be observed, indicating that the electrons can transfer from $\mathrm{SrTiO}_{3}$ to $\mathrm{MoSe}_{2}$. Two peaks of $\mathrm{Sr} 3 \mathrm{~d}$ in Fig. 5(b) at $133.7 \mathrm{eV}$ and $135.3 \mathrm{eV}$ are assigned to 

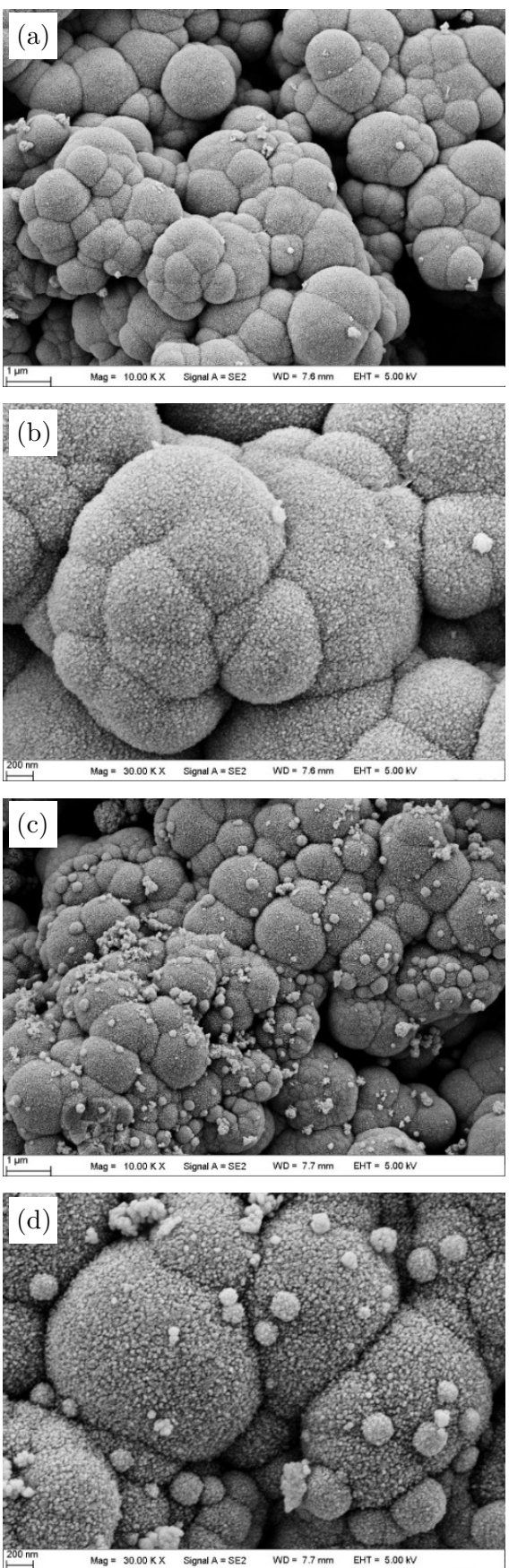

(e)

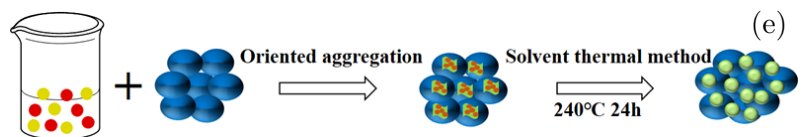

Fig. 2. (a) and (b) SEM images of $\mathrm{SrTiO}_{3}$, (c) and (d) SEM images of S3 and (e) formation mechanism of S1-S7.

Sr $3 \mathrm{~d}_{5 / 2}$ and $\mathrm{Sr} 3 \mathrm{~d}_{3 / 2},{ }^{22}$ and the binding energies corresponding to Ti $2 \mathrm{p}_{3 / 2}$ and $\mathrm{Ti} 2 \mathrm{p}_{1 / 2}$ are $458.1 \mathrm{eV}$ and $463.9 \mathrm{eV}$ in Fig. 5(c). ${ }^{23}$ The binding energy of $\mathrm{O}$ $1 \mathrm{~s}$ is $531.8 \mathrm{eV}$ in Fig. 5(d). ${ }^{24}$ In Fig. 5(e), the binding
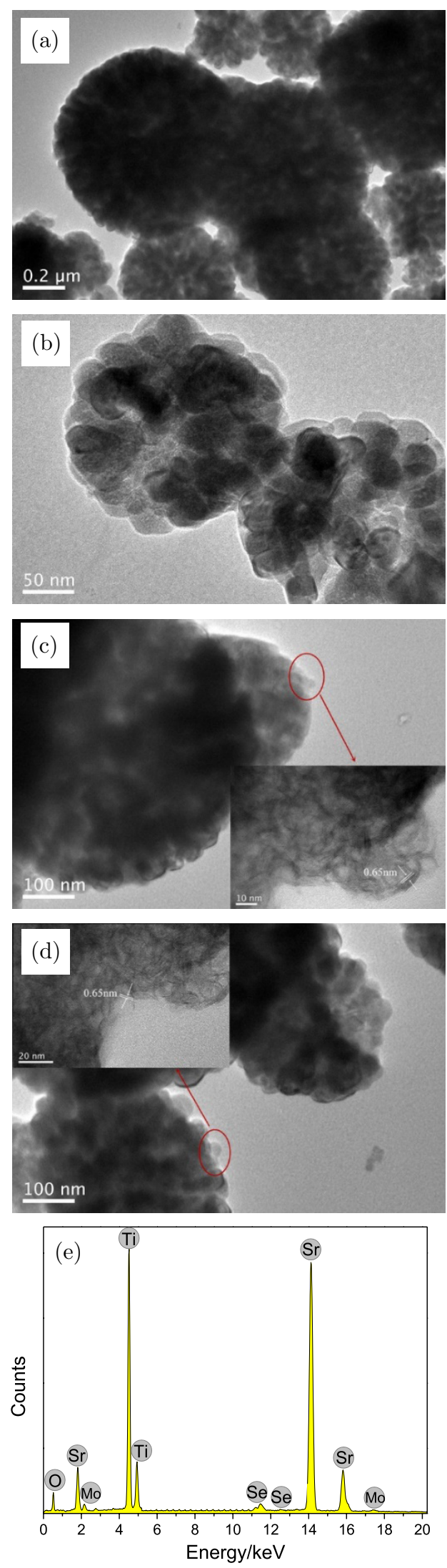

Fig. 3. (a) and (b) TEM images of $\mathrm{SrTiO}_{3}$, (c) and (d) HRTEM images of S3 and (e) EDS of S3. 


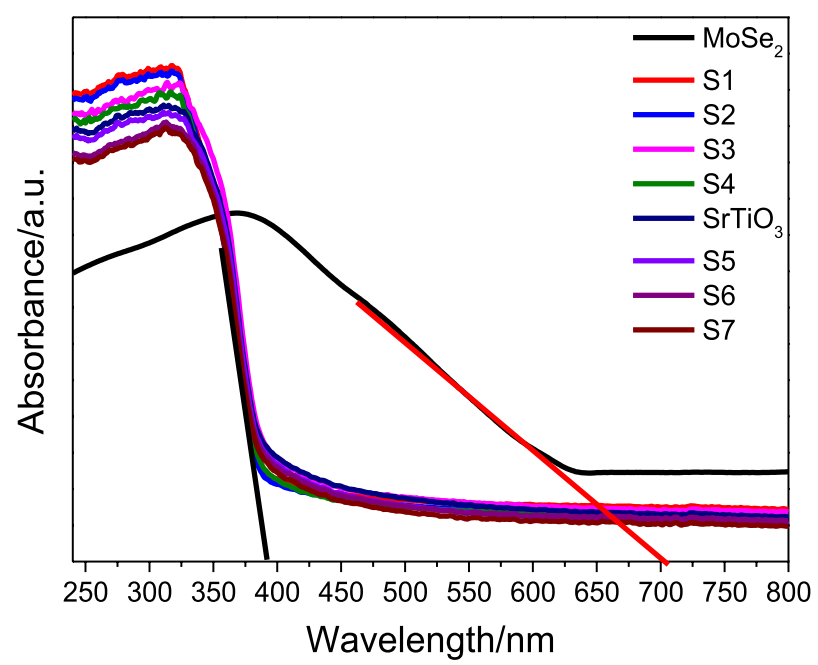

Fig. 4. UV-Vis absorption spectra of $\mathrm{SrTiO}_{3}, \mathrm{~S} 1, \mathrm{~S} 2, \mathrm{~S} 3, \mathrm{~S} 4$, S5, S6, S7 and $\mathrm{MoSe}_{2}$ samples.

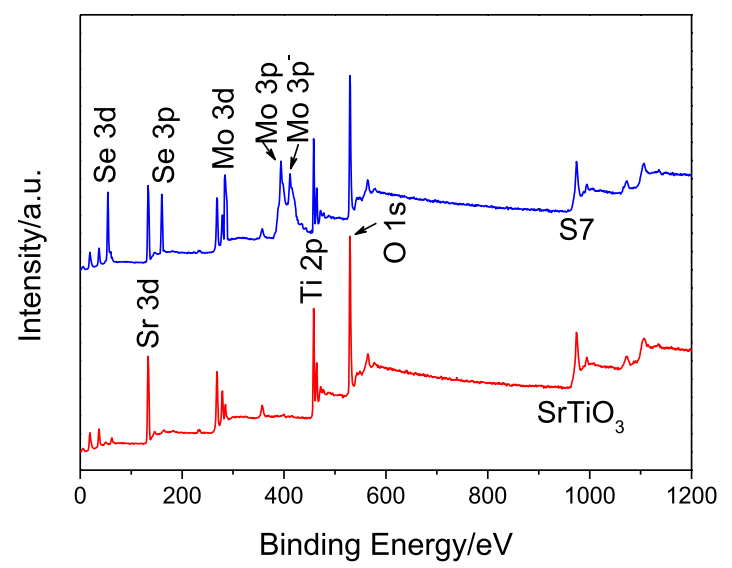

(a)

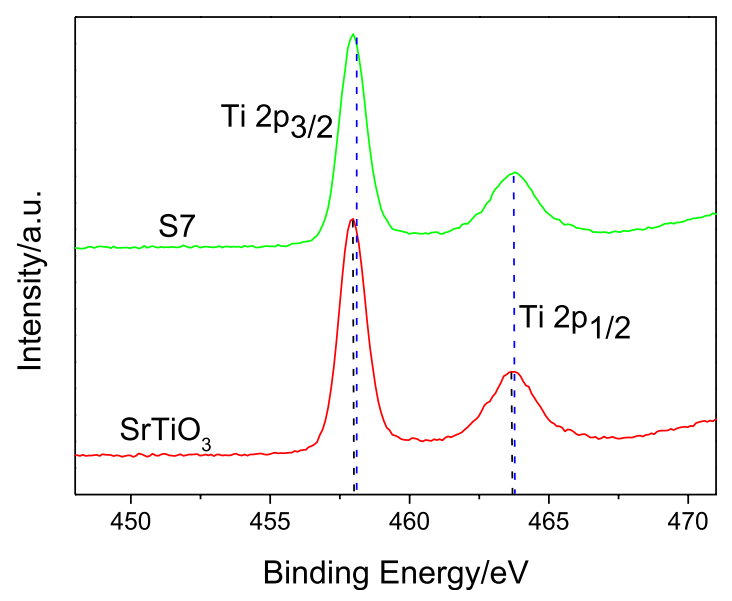

(c) energies of Mo $3 \mathrm{~d}_{5 / 2}$ and Mo $3 \mathrm{~d}_{3 / 2}$ peaks are located at $229.1 \mathrm{eV}$ and $232.3 \mathrm{eV},{ }^{25}$ respectively, suggesting that $\mathrm{Mo}^{4+}$ exists in the sample. Figure $5(\mathrm{f})$ shows a high-resolution XPS spectrum of Se 3d. The peak is split into two well-defined peaks at $54.5 \mathrm{eV}$ and $55.4 \mathrm{eV}$, corresponding to the Se $3 \mathrm{~d}_{5 / 2}$ and Se $3 \mathrm{~d}_{3 / 2}$ peaks, ${ }^{26}$ indicating that $\mathrm{Se}^{2-}$ exists in the sample. The ratio of atomic percentage of Mo and Se analyzed by XPS was 11.32:21.70, which is close to 1:2. In summary, the presence of $\mathrm{MoSe}_{2}$ and $\mathrm{SrTiO}_{3}$ in the $\mathrm{S} 7$ sample was further confirmed by the high resolution of the XPS spectrum, which is consistent with XRD and TEM analysis.

As indicated in Fig. 6, the degradation performance under UV light is better than that under visible light. Judging from Fig. 6(a), the MO has not been completely degraded after 75 min reaction,

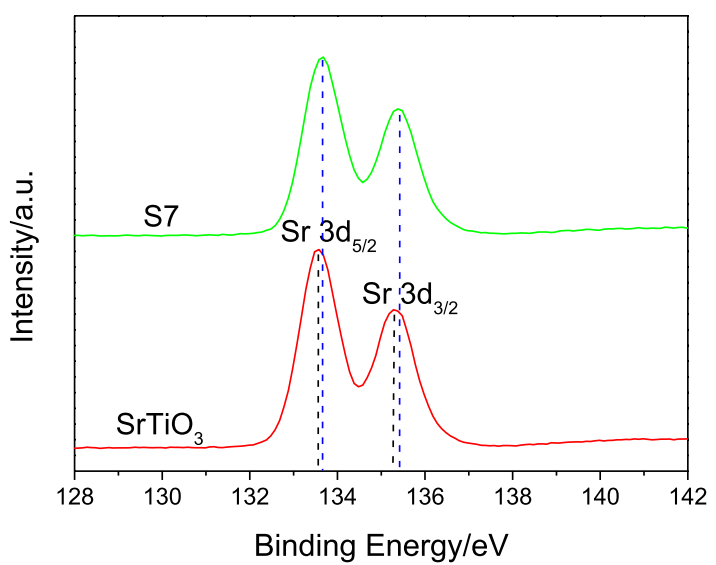

(b)

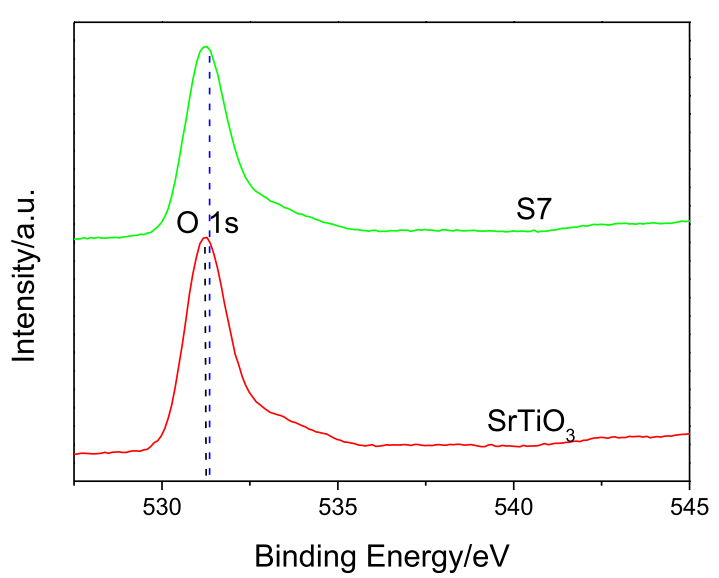

(d)

Fig. 5. (a) XPS survey spectra of sample $\mathrm{SrTiO}_{3}$ and S7, (b-f) high resolution XPS spectra of Sr, Ti, O, Mo and Se. 


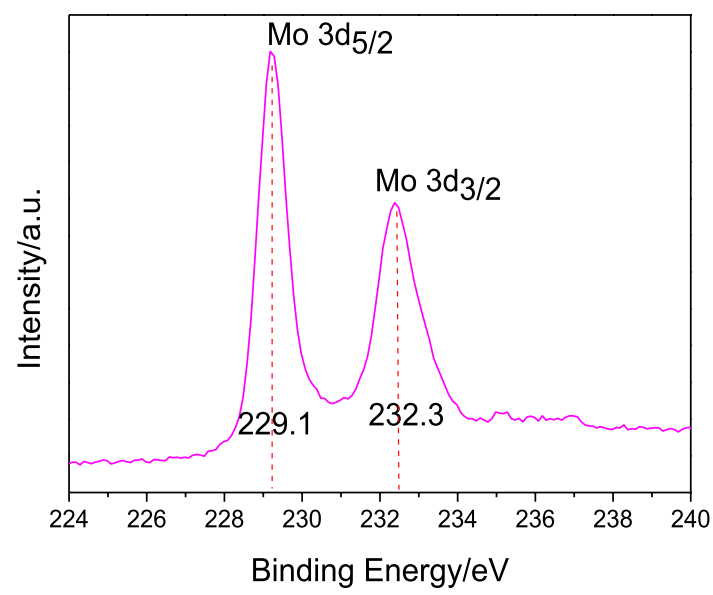

(e)

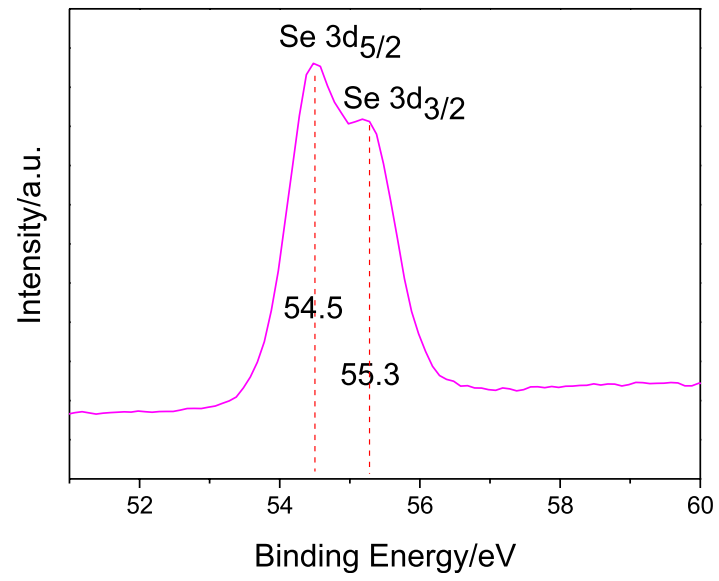

(f)

Fig. 5. (Continued)

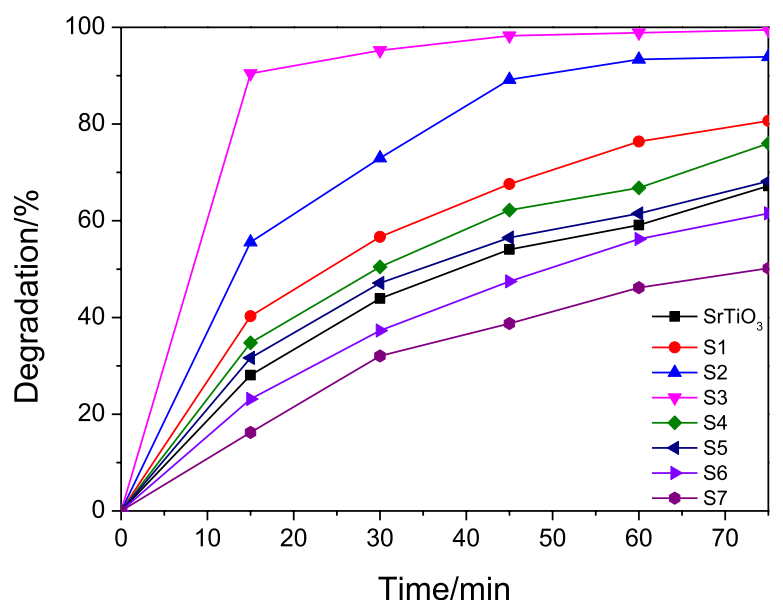

(a)

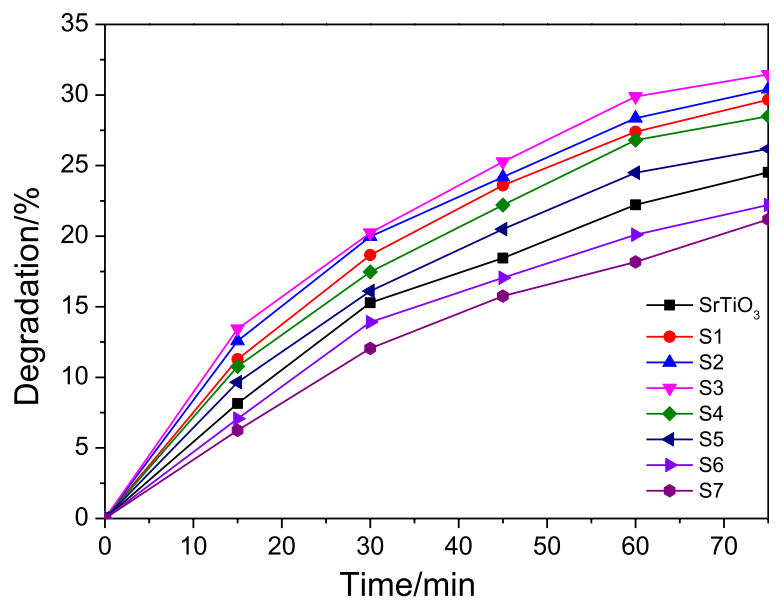

(b)

Fig. 6. The degradation performance of pure $\mathrm{SrTiO}_{3}$ and $\mathrm{S} 1$, $\mathrm{S} 2$, S3, S4, S5, S6, S7 in $75 \mathrm{~min}$. (a) under UV light (b) under visible light. indicating the relative poor activities of all $\mathrm{SrTiO}_{3}$ based catalysts. Comparing samples $\mathrm{S} 1-\mathrm{S} 5$ with pure $\mathrm{SrTiO}_{3}$, the degradation rate is higher for S1-S5. Therefore, loading $\mathrm{MoSe}_{2}$ can improve the photocatalytic activity of $\mathrm{SrTiO}_{3}$. Moreover, S3 sample with 0.1 wt.\% loading demonstrated highest activity, with the degradation rate of $99.46 \%$. This is because small amount of $\mathrm{MoSe}_{2}$ caused an efficient charge separation of $\mathrm{SrTiO}_{3}$, which suppressed the electron-hole recombination. However, when the $\mathrm{MoSe}_{2}$ loading increased from $0.1 \mathrm{wt} . \%$ to $5 \mathrm{wt} . \%$ (S3-S7), the MO degradation rate decreased gradually. The major reason is that a large amount of $\mathrm{MoSe}_{2}$ grew on the surface of $\mathrm{SrTiO}_{3}$, covering a part of the catalytic activity center, and blocking the UV light needed in the reaction process with the increase of $\mathrm{MoSe}_{2}$ content.

The intensity of the characteristic peaks in the UV-Vis absorption spectra of the MO concentrations is different, so the photocatalytic activity of the corresponding different catalysts can be evaluated. The higher the concentration of MO is, the stronger the peak is. In Fig. 7, the maximum absorption peak at $460 \mathrm{~nm}$ in the UV-Vis absorption spectra is the weakest after the degradation of MO solution for 45 min when S3 acted as the catalyst under UV irradiation. The peak intensity (Fig. 7) corresponds to the change of the degradation rate [Fig. 6(a)].

The stability and reusability of photocatalysts are important factors for its application. Herein, the stability of the $0.1 \mathrm{wt} . \% \mathrm{MoSe}_{2} / \mathrm{SrTiO}_{3}(\mathrm{~S} 3)$ is investigated. After six cycles of the photodegradation 


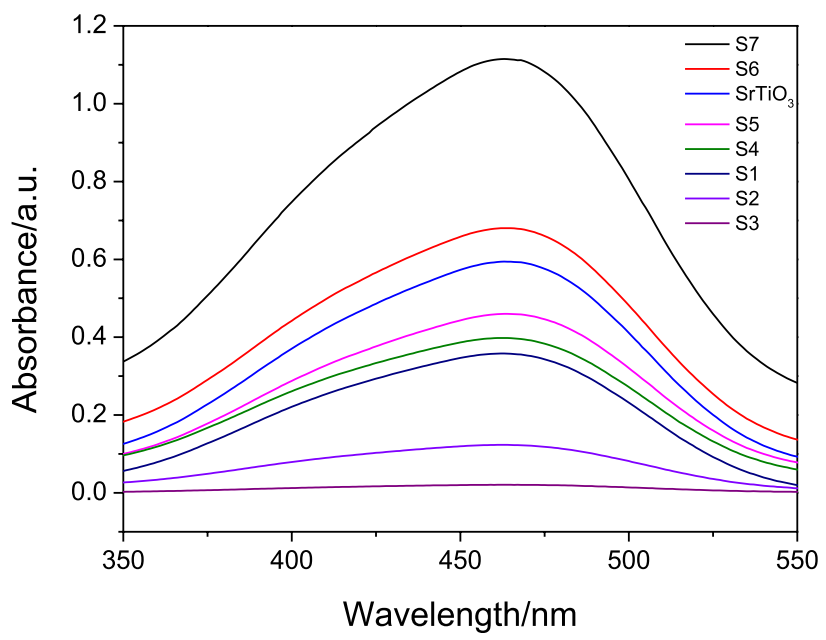

Fig. 7. UV-Vis absorption of $\mathrm{MO}$ with the variation of $\mathrm{MoSe}_{2}$ loading at $45 \mathrm{~min}$ irradiation time under UV irradiation.

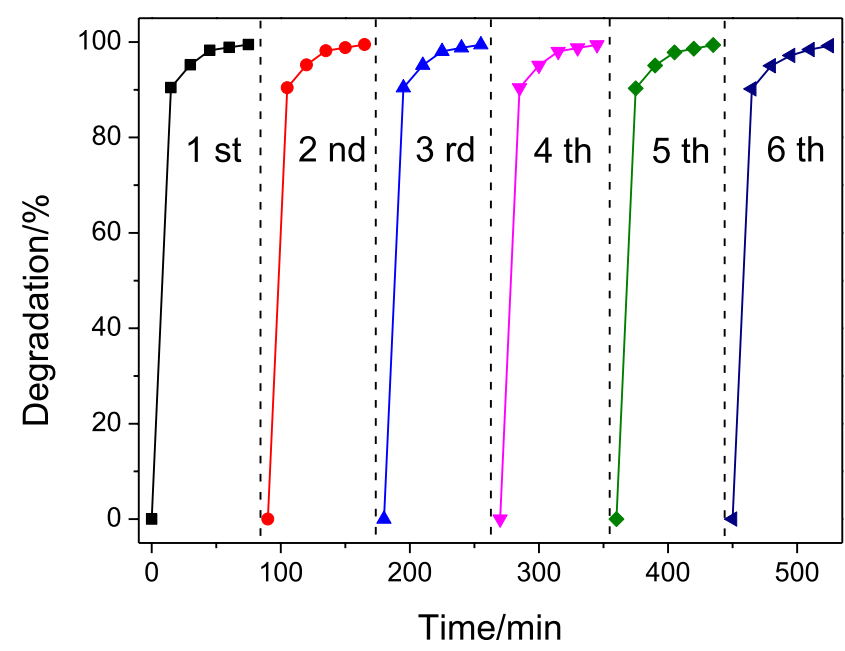

Fig. 8. Recyclability of photodegradation of MO over S3 under UV irradiation for $75 \mathrm{~min}$.

of MO, no significant loss in photocatalytic activity is observed, as shown in Fig. 8, indicating the excellent stability of $\mathrm{MoSe}_{2} / \mathrm{SrTiO}_{3}$ catalyst.

As shown in Fig. 9, the degradation rate of $\mathrm{MO}$ was not significantly reduced after addition of $\mathrm{IPA},{ }^{27}$ indicating that $\cdot \mathrm{OH}$ was not an important contributor to the degradation process. In contrast, the degradation rate of MO decreased significantly after adding TEOA and BQ into MO solution, ${ }^{28,29}$ indicating that $\mathrm{h}^{+}$was trapped by TEOA and $\cdot \mathrm{O}_{2}^{-}$ was captured by BQ. Thus, $\mathrm{h}^{+}$and $\cdot \mathrm{O}_{2}^{-}$were the main active substances of the $\mathrm{S} 3$ catalyst under UV irradiation.

The valence band and conduction band of $\mathrm{MoSe}_{2}$ and $\mathrm{SrTiO}_{3}$ can be calculated according to the

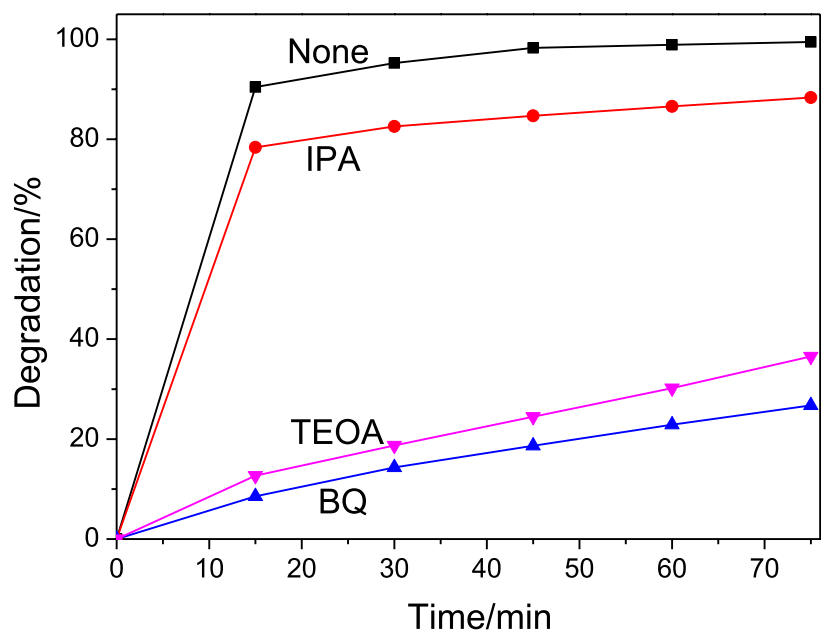

Fig. 9. Photodegradation of MO using different radical scavenger over S3. (a) no scavenger under $\mathrm{O}_{2}$-saturated condition, (b) IPA for $\cdot \mathrm{OH}$ under $\mathrm{O}_{2}$-saturated condition, (c) TEOA for $\mathrm{h}^{+}$under $\mathrm{O}_{2}$-saturated condition, (d) BQ for $\cdot \mathrm{O}_{2}^{-}$under $\mathrm{O}_{2^{-}}$ saturated condition.

formula of valence band energy $E_{\mathrm{VB}}$ and conduction band energy $E_{\mathrm{CB}}{ }^{30-32}$ :

$$
\begin{aligned}
& E_{\mathrm{VB}}=X-E^{c}+0.5 E_{g}, \\
& E_{\mathrm{CB}}=X-E^{c}-0.5 E_{g},
\end{aligned}
$$

where $X$ represents the geometrical mean of the absolute electronegativity of each atom in the semiconductor, $E^{c}$ represents the electron free energy (in general $4.5 \mathrm{eV}$ ), $E_{g}$ represents the semiconductor bandgap energy. $E_{g}\left(\mathrm{SrTiO}_{3}\right)=3.2 \mathrm{eV}$, $E_{g}\left(\mathrm{MoSe}_{2}\right)=1.75 \mathrm{eV}$ have been calculated from Fig. 4. From the table, $X\left(\mathrm{SrTiO}_{3}\right)=4.95, \quad X$ $\left(\mathrm{MoSe}_{2}\right)=5.13$ are obtained according to calculation. So final results are $E_{\mathrm{VB}}\left(\mathrm{SrTiO}_{3}\right)=2.05 \mathrm{~V}$, $E_{\mathrm{CB}}\left(\mathrm{SrTiO}_{3}\right)=-1.15 \mathrm{~V}, \quad E_{\mathrm{VB}}\left(\mathrm{MoSe}_{2}\right)=1.51 \mathrm{~V}$, $E_{\mathrm{CB}}\left(\mathrm{MoSe}_{2}\right)=-0.24 \mathrm{~V}$. To this end we can propose the following mechanism (Fig. 10):

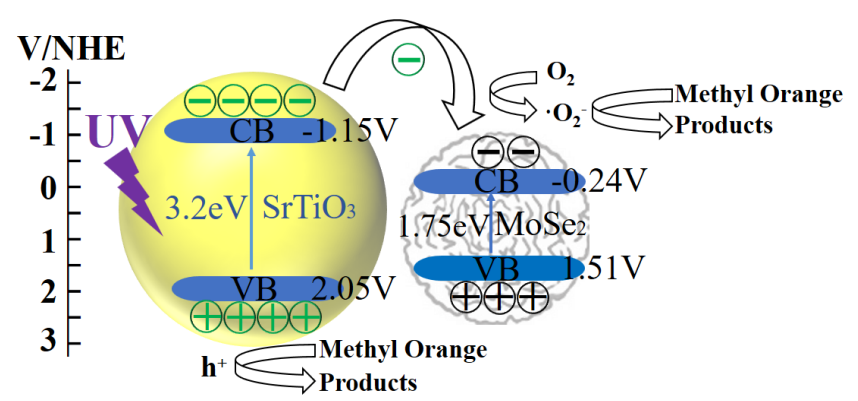

Fig. 10. The proposed mechanism of the charge transfer and separation process for the $\mathrm{MoSe}_{2} / \mathrm{SrTiO}_{3}$ heterostructures under UV irradiation. 
The conduction band position of $\mathrm{SrTiO}_{3}$ is higher than that of $\mathrm{MoSe}_{2}$, the electrons can easily transfer from $\mathrm{SrTiO}_{3}$ to $\mathrm{MoSe}_{2}$. Under UV light irradiation, the photo-excited electrons $\mathrm{VB}$ of $\mathrm{SrTiO}_{3}$ are directly transferred to $\mathrm{CB}$ of $\mathrm{SrTiO}_{3}$, creating holes in the VB. ${ }^{33}$ The photo-generated electrons and holes are inclined to recombine quickly without $\mathrm{MoSe}_{2}$ loading, causing the low photocatalytic activity. However, after introducing $\mathrm{MoSe}_{2}$, electrons in the $\mathrm{CB}$ of $\mathrm{SrTiO}_{3}$ will migrate to the $\mathrm{MoSe}_{2}$, which makes the electrons more mobile and provides highly active sites for the photocatalysis process. In addition, the holes left in the $\mathrm{SrTiO}_{3}$ can be consumed by the MO dyes. Besides, $\mathrm{O}_{2}$ receives excess electrons from $\mathrm{MoSe}_{2}$ and combines it into $\cdot \mathrm{O}_{2}^{-}$. As we all know, $\mathrm{O}_{2}^{-}$has a strong oxidizing property. Thus, the above-described mechanism can effectively suppress the recombination between electrons and holes, enhance interfacial charge transfer, lengthen the lifetime of charge carriers and enlarge the active sites.

\section{Conclusions}

In summary, the $\mathrm{MoSe}_{2} / \mathrm{SrTiO}_{3}$ heterostructures were prepared by the two-step hydrothermal method for the first time in the degradation of MO under UV light and visible light irradiation. It was found that the degradation rate of MO under UV irradiation was much higher than that under visible light. Under UV irradiation, the optimum loading of $\mathrm{MoSe}_{2}$ is $0.1 \mathrm{wt} . \%$. At $75 \mathrm{~min}$, the degradation rate of MO reaches $99.46 \%$, which is much higher than pure $\mathrm{SrTiO}_{3}$. However, excessive loading can hinder the active center, affecting the process of photodegradation. The proposed mechanism shows that the proper amount of $\mathrm{MoSe}_{2}$ can effectively suppress the recombination of electrons and holes in $\mathrm{SrTiO}_{3}$, enhance the movement of interfacial electrons, prolong the time of electrons and increase the photocatalytic activity center. At the same time, $\cdot \mathrm{O}_{2}^{-}$generation also plays a significant role. Therefore, the development of cheap nonprecious metal composites for photodegradation has great potential because of its high economic and catalytic activity.

\section{Acknowledgments}

This work was financially supported by Top-notch Academic Programs Project of Jiangsu Higher Education Institutions (PPZY2015B145); and Industry
Supporting Project of Zhenjiang of Jiangsu Province (GY2014037).

\section{References}

1. M. Prathap Kumar, G. A. Suganya Josephine and A. Sivasamy, J. Mol. Liq. 242, 789 (2017).

2. M. Kubicek, A. H. Bork and J. L. M. Rupp, J. Mater. Chem. A 5, 11983 (2017).

3. J. Zhu, H. Li, L. Zhong, P. Xiao, X. Xu, X. Yang, Z. Zhao and J. Li, ACS Catal. 4, 2917 (2014).

4. D. Singh, R. Singh, S. Sharma and M. Sharma, Ionics 19, 499 (2012).

5. S.-W. Baek, J. H. Kim and J. Bae, Solid State Ionics 179, 1570 (2008).

6. R. A. De Souza, Adv. Funct. Mater. 25, 6326 (2015).

7. Z. Wu, Y. Zhang, X. Wang and Z. Zou, New J. Chem. 41, 5678 (2017).

8. S. Cheng, Y. Wang, L. Zhuang, J. Xue, Y. Wei, A. Feldhoff, J. Caro and H. Wang, Angew. Chem. 55, 10895 (2016).

9. J. H. Liu, L. Zhang, N. X. Li, Q. W. Tian, J. C. Zhou and Y. M. Sun, J. Mater. Chem. A 3, 706 (2015).

10. Q. Tian, L. Zhang, J. Liu, N. Li, Q. Ma, J. Zhou and Y. Sun, RSC Adv. 5, 734 (2015).

11. J. Kong, Z. Rui, S. Liu, H. Liu and H. Ji, Chem. Commun. 53, 12329 (2017).

12. Z. Zhang, W. Jing, X. Tian, T. Yu and J. Ma, J. Mater. Sci. 53, 6170 (2018).

13. J. Kong, Z. Rui and H. Ji, Ind. Eng. Chem. Res. 56, 9999 (2017).

14. H. Tang, H. Huang, X. S. Wang, K. Q. Wu, G. G. Tang and C. S. Li, Appl. Surf. Sci. 379, 296 (2016).

15. D. J. Sathe, P. A. Chate, P. P. Hankare, A. H. Manikshete and A. S. Aswar, J. Mater. Sci-Mater El. 24, 438 (2012).

16. D. Kong, H. Wang, J. J. Cha, M. Pasta, K. J. Koski, J. Yao and Y. Cui, Nano Lett. 13, 1341 (2013).

17. J. C. Shaw, H. Zhou, Y. Chen, N. O. Weiss, Y. Liu, Y. Huang and X. Duan, Nano Res. 7, 511 (2015).

18. H. Tang, K. Dou, C.-C. Kaun, Q. Kuang and S. Yang, J. Mater. Chem. A 2, 360 (2014).

19. L. F. da Silva, J.-C. M'Peko, J. Andrés, A. Beltrán, L. Gracia, M. I. B. Bernardi, A. Mesquita, E. Antonelli, M. L. Moreira and V. R. Mastelaro, J. Phys. Chem. C 118, 4930 (2014).

20. A. Ramasubramaniam, Phys. Rev. B 86, 2757 (2012).

21. S. Deng, Y. Zhong, Y. Zeng, Y. Wang, Z. Yao, F. Yang, S. Lin, X. Wang, X. Lu, X. Xia and J. Tu, Adv. Mater. 29, 1700748 (2017).

22. J. Luo and P. A. Maggard, Adv. Mater. 18, 514 (2006).

23. J. Kou, J. Gao, Z. Li, H. Yu, Y. Zhou and Z. Zou, Catal. Lett. 145, 640 (2014). 
24. H. Tan, Z. Zhao, W. B. Zhu, E. N. Coker, B. Li, M. Zheng, W. Yu, H. Fan and Z. Sun, ACS Appl. Mater. Interfaces 6, 19184 (2014).

25. W. Zhou, Z. Yin, Y. Du, X. Huang, Z. Zeng, Z. Fan, H. Liu, J. Wang and H. Zhang, Small 9, 140 (2013).

26. B. B. Wang, M. K. Zhu, I. Levchenko, K. Zheng, B. Gao, S. Xu and K. Ostrikov, J. Cryst. Growth 475, 1 (2017).

27. P. Lisowski, J. C. Colmenares, O. Mašek, W. Lisowski, D. Lisovytskiy, A. Kamińska and D. Łomot, ACS Sustain. Chem. Eng. 5, 6274 (2017).

28. S. Liu, N. Zhang, Z. R. Tang and Y. J. Xu, ACS Appl. Mater. Interfaces 4, 6378 (2012).
29. Q. Liang, J. Jin, M. Zhang, C. Liu, S. Xu, C. Yao and Z. Li, Appl. Catal. B-Environ. 218, 545 (2017).

30. I. Aslam, C. Cao, M. Tanveer, M. H. Farooq, W. S. Khan, M. Tahir, F. Idrees and S. Khalid, $R S C A d v$. 5, 6019 (2015).

31. J. Jin, J. Yu, D. Guo, C. Cui and W. Ho, Small 11, $5262(2015)$.

32. N. Li, H. Teng, L. Zhang, J. Zhou and M. Liu, RSC Adv. 5, 95394 (2015).

33. C. Karunakaran, S. SakthiRaadha, P. Gomathisankar and P. Vinayagamoorthy, Dalton Trans. 42, 13855 (2013). 